\title{
Für eine Welt ohne Atomwaffen - Für eine Zukunft
}

\section{Knüsli ${ }^{a}$, A. Nidecker ${ }^{b}$}

a Dr. med., Präsident PSR / IPPNW Schweiz

b Prof. Dr. med., OK-Präsident IPPNW Weltkongress 2010
Korrespondenz:

Prof. Dr. med. A. Nidecker

Röntgeninstitut IMAMED

Rheinstrasse 16

CH-4410 Liestal

andreas.nidecker@imamed.ch,

anidecker@bluewin.ch

\section{PSR/IPPNW-Weltkongress 2010 in Basel}

Die Schweizer Sektion der PSR/IPPNW (Physicians for Social Responsibility/International Physicians for the Prevention of Nuclear War ÄrztInnen für Soziale Verantwortung/Internationale ÄrztInnen zur Verhütung des Atomkriegs, Friedensnobelpreis 1985) hat die Zusage für die Durchführung des nächsten Weltkongresses erhalten. Dieser wird vom 25. bis 29. August 2010 in Basel stattfinden. Der 25. und 26. August sind für den Studentenkongress reserviert und vom 27. bis 29. August wird der Hauptkongress durchgeführt werden.

\section{Hintergrund}

Die PSR/IPPNW Schweiz wurden 1981 von Dr. Martin Vosseler in Bern gegründet. Unsere Organisation engagiert sich im Interesse einer sicheren Zukunft für unsere Kinder und die folgenden Generationen gegen die nukleare Proliferation. So unrealistisch dies momentan auch erscheinen mag, so entschieden verfolgen wir das Ziel einer globalen atomaren Abrüstung. Wir wissen, dass wir damit den politischen Absichten der Schweiz und dem Empfinden der meisten Schweizerinnen und Schweizer entsprechen und im Sinne vieler Menschen in Europa und der übrigen Welt handeln.

Seit der Gründung unserer Nichtregierungsorganisation, die weltweit über 200000 Mitglieder in fast 60 Ländern zählt, verfolgt PSR/IPPNW eine aktive Friedenspolitik. Dies ist heute aktueller den je: Länder im Nahen Osten werden verdächtigt, unter dem Vorwand der Propagierung der friedlichen Nutzung der Atomenergie insgeheim Atomwaffen zu entwickeln. Präsidenten europäischer Atommächte posieren vor neuesten Atom-U-Booten und trotz Atomsperrvertrag werden bereits Atomwaffen der vierten Generation entwickelt.

Die Bedrohung der globalen Sicherheit durch Atomwaffen ist für uns eine zutiefst ärztliche Frage: Wie können wir zusehen, wenn bei einem atomaren Schlag Hunderttausende Menschenleben ausgelöscht werden, wie dies 1945 geschehen ist? Prävention ist vorrangig - ärztliche Hilfe im Falle eines Atomkrieges illusorisch. Der
Glaube der Atommächte, Atomwaffen könnten weltweite Sicherheit garantieren, muss erst recht hinterfragt werden, da die Bedrohung der Menschen und ihres Habitats heute vielmehr durch Naturereignisse infolge des Klimawandels, ökologische Katastrophen oder terroristische Aktionen zustande kommt als durch Kriege zwischen Nationen.

Im Jahre 2010 wird der atomare Nicht-Weiterverbreitungsvertrag (NPT $=$ Non Proliferation Treaty) unter den Nationen neu diskutiert werden. Die NPT Review Conference ist ein Ereignis von grösster Bedeutung für die kommenden Generationen. Die Ländergemeinschaft steht vor der Frage, ob die Welt weiterhin geteilt werden kann in Staaten mit Anrecht auf Atomwaffen und solche ohne. Auch renommierte Politiker wie Henry Kissinger teilen heute die Meinung mit PSR/IPPNW: Eine echte allgemeine Abrüstung auch der Atomwaffenstaaten ist der einzig erfolgversprechende Weg zu einer stabileren Welt. In einer Zeit der Ressourcenknappheit stehen ferner die Ausgaben für Atomwaffen in keinem Verhältnis zu den Aufwendungen für soziale Anliegen und Bedürfnisse im Gesundheits- und Erziehungswesen.

\section{Austragungsort}

Die Schweiz hat als neutraler Staat immer wieder eine Vermittlerrolle übernommen und ist dank ihrer humanitären Tradition prädestiniert für friedenspolitische Aktivitäten. Die Basler Universität ist die älteste der Schweiz und feiert 2010 ihr 550-Jahre-Jubiläum. In humanistischer Tradition haben hier unter anderem der Mediziner Paracelsus, die Mathematiker Jacob und Johann Bernoulli, der Philosoph Carl Jaspers, der Theologe Karl Barth und der Zoologe Adolf Portmann geforscht und gelehrt. Als Austragungsort ist uns vom Rektor der Universität Basel das Kollegiengebäude angeboten worden, wofür wir sehr dankbar sind.

Der Kongress dauert traditionellerweise sechs Tage, mit einem zweitägigen Studentenkongress, der dem dreitägigen Hauptkongress vorangeht. Das Businessmeeting der Organisation folgt am sechsten Tag. 


\section{Kongressthemen}

Der Kongress steht unter dem Titel: For a Nuclear Weapon Free World - For a Future. Die Hauptthemen werden in Plenarsitzungen besprochen. Zusätzlich finden kleinere Sitzungen und Workshops zu ökologischen, friedens- und entwicklungspolitischen Fragen statt. Folgende Themen stehen im Vordergrund:

- die 2010 stattfindende NPT Review Conference in New York;

- die aktuelle Situation bezüglich atomarer Abrüstung der traditionellen Atommächte;

- die Aufrüstung der jungen nach Atomwaffen strebenden Nationen;

- die Chancen für atomwaffenfreie Zonen in Europa und im Mittleren Osten;

- die nukleare Verseuchung durch die biosphärischen Atomwaffenversuche in den 40er und 50er Jahren.

Weitere Diskussionsgebiete werden sein:

- die radioaktive Verstrahlung nach der Katastrophe von Tschernobyl und anderen AKWUnfällen;

- die Urangewinnung und ihre gesundheitlichen Folgen bei der Bevölkerung in den Produktionsländern;

- Fragen um die Auslegung des Internationalen nuklearen Rechts;

- der Wissensstand um die radioaktive Niedrigstrahlung;

- die Diskussion neuer epidemiologischer Forschungsresultate.

\section{Zielgruppen}

Der Kongress wird interdisziplinär sein, richtet sich aber in erster Linie an Ärztinnen und Ärzte sowie Verantwortliche im Gesundheitswesen und Studenten. Wir erwarten etwa 600-800 Ärztinnen und Ärzte aus der ganzen Welt und zusätzlich bis zu 300 Medizinstudenten. Die Bevölkerung in der Triregio werden wir durch öffentliche Anlässe auf unser Anliegen aufmerksam machen.

\section{Aufruf zur Unterstützung und Mitarbeit}

Das Budget der PSR/IPPNW Schweiz ist limitiert. Die Teilnahmegebühren decken die Auslagen bei weitem nicht, wobei wir mit Gesamtkosten von 350000 Franken rechnen. Wir sind für die Finanzierung des IPPNW-Weltkongresses auf Unterstützung durch Spender und Sponsoren angewiesen. Gerade Teilnehmende aus Afrika und Osteuropa sind auf unsere Unterstützung bezüglich der Reise- und Unterkunftskosten angewiesen.

Wir suchen nun Kolleginnen und Kollegen, die unsere Sorge teilen und sich im Hinblick auf den kommenden Kongress für eine atomwaffenfreie Welt für unsere Kinder einsetzen möchten. Natürlich würden wir uns freuen über Neumitglieder oder «Wiedereinsteiger».

\section{Information:}

Sekretariat PSR/IPPNW Schweiz, Tel. 061

27150 25, E-Mail: sekretariat@ippnw.ch, Internet: www.ippnw.ch 